\title{
Molecular Cloning and Functional Expression of cDNAs Encoding Oxidosqualene Cyclases from Costus speciosus
}

\author{
Noriaki Kawano, Koji Ichinose, and Yutaka EBIZuKA* \\ Graduate School of Pharmaceutical Sciences, The University of Tokyo, Hongo, Bunkyo-ku, Tokyo 113-0033, Japan. \\ Received November 5, 2001; accepted December 10, 2001
}

\begin{abstract}
Costus speciosus produces a large quantity of steroidal glycosides derived from the sole aglycone, diosgenin. Cycloartenol, a product of oxidosqualene cyclase (OSC), is postulated to be a common intermediate for phytosterols of primary metabolism and diosgenin of secondary metabolism, possibly providing a metabolic branch point. Two cDNAs, CSOSC1 and CSOSC2, were cloned from $C$. speciosus by RT-PCR and cDNA library screening. Both cDNAs encode 759 amino acids with high mutual identity $(74 \%)$, resembling $(>55 \%$ identity) the known OSCs. Phylogenetic tree analysis indicated that the gene products occupy distinct positions from those of cycloartenol synthases (CASs) and triterpene synthases from dicotyledonous plants. By functional expression in yeast, CSOSC1 and CSOSC2 were proved to encode a CAS and a multifunctional triterpene synthase, respectively. The present result is the first demonstration of the functional expression of OSCs from monocotyledonous plants.
\end{abstract}

Key words cDNA cloning; 2,3-oxidosqualene cyclase; monocotyledonous; Costus speciosus; cycloartenol; triterpene

Steroidal glycosides occur in a wide range of plant species, mostly as spirostane derivatives containing oligosaccharide moieties attached at C-3. ${ }^{1)}$ They are known as steroid saponins of characteristic antifungal and hemolytic activities.

Costus speciosus (Koenig.) Sm. (Costaceae) is a tropical to subtropical monocotyledonous plant that contains a large amount of spirostanol glycosides (SGs). Diosgenin is the sole aglycone of these glycosides ${ }^{2)}$ in contrast to the structural heterogeneity of sapogenins shown by other steroid saponin-producing plants. ${ }^{3)}$ In addition, it is notable that no cyclic triterpenes have been reported from this plant.

Two major SGs of this plant, dioscin and gracillin, are known to be accumulated in the rhizome of field grown plants, ${ }^{4)}$ while they are found in whole parts of in vitro cultured plantlets. ${ }^{5)}$ With regard to their biosynthesis, very little is known except for the final conversion step from furostanol glycosides (FGs) to SGs, which is catalyzed by a specific glycosidase, furostanol glycoside $26-O-\beta$-glucosidase. ${ }^{5-9)}$ The early stage of diosgenin biosynthesis is assumed to partially share the same pathway with phytosterols, such as $\beta$ sitosterol, campesterol, and stigmasterol of primary metabolism.

Earlier feeding experiments using ${ }^{14} \mathrm{C}$ - and ${ }^{3} \mathrm{H}$-labeled precursors provided the following observations in SG biosynthesis: (a) cholesterol and $\beta$-sitosterol are incorporated ${ }^{10-14)}$; (b) the cholesterol side chain undergoes three hydroxylations at $\mathrm{C}-26,16$, and 22 in that order $^{15,16)}$; (c) cholesterol derives from mevalonate. ${ }^{17)}$ It is of great interest to know whether the cycloartenol pool is common to phytosterol and SG biosynthesis. Cycloartenol is elaborated from an acyclic substrate, 2,3-oxidosqualene, by cycloartenol synthase (CAS), an oxidosqualene cyclase (OSC, EC 5.4.99.-) under strict stereochemical control. ${ }^{18)}$ OSCs are ubiquitous in nature, and those from the plant kingdom constitute two subtypes with respect to their catalytic mechanism. Steroidgenic OSCs, including cycloartenol synthase (CAS), cyclize 2,3-oxidosqualene folded in pre chair-boat-chair conformation, whereas allchair conformation is involved in most of the other triterpene-producing OSCs. More than eighty different triterpene skeletons have been reported from higher plants, and often found is the co-occurrence of more than one skeletal type of triterpene in one plant species. Recent cloning of multifunctional synthases producing multiple products raises a question as to the occurence of the corresponding number of product specific synthases.

A number of plant OSCs have been characterized by cDNA cloning and their functional expression in yeast. Examples include CAS cDNAs from Arabidopsis thaliana $(C A S 1),{ }^{19)}$ Panax ginseng $(P N X),{ }^{20)}$ Pisum sativum $(C A S P E A)^{21)}$ and Glycyrrhiza glabra $(G g C A S 1){ }^{22)}$ nine cDNAs encoding product specific triterpene synthases: $\beta$ amyrin synthases from Panax ginseng (PNY and PNY2), ${ }^{20,23)}$ P. sativum $(P S Y)^{24)}$ and G. glabra $(G g b A S 1)^{25)}$; and two lupeol synthases from Olea europaea $(O E W)^{26)}$ and Taraxacum officinale $(T R W){ }^{26)}$ In addition, apparent multifunctional triterpene synthase cDNAs have been identified from $P$. sativum $(P S M)^{24)}$ and A. thaliana (LUP1 and YUP8H12R.43). ${ }^{27-30)}$ So far, the functionally characterized OSCs of plant origin are all derived from dicotyledonous (dicot) species, whereas monocotyledonous (monocot) examples are limited to the two putative cDNAs: Allium macrostemon (ALLOSC1), ${ }^{31)}$ Oryza sativa (OSCAS1, accession number AF169966).

The present study deals with molecular cloning and functional expression of cDNAs encoding C. speciosus OSCs. This is the first demonstration of the functional expression of OSCs from monocot plants.

\section{MATERIALS AND METHODS}

Materials and Strains Plantlets of C. speciosus were cultured in vitro as described previously. ${ }^{5}$ Escherichia coli strains were NovaBlue (Novagen, supE44 hsdE44 hsdR17 recAl endA1 gyr96 thi-1 relAl lac $\left[F^{\prime}\right.$ proAB+ lacIq lacZAM15::Tn 10]), and DH5 $\alpha$ (supE44 lacU169 [ $\phi 80 l a c Z \Delta M 15]$ hsdR17 recAl gyrA96thi-1 relA1). The yeast strain was Saccharomyces cerevisiae GIL77 (gal2 hem3-6 erg7 ura3-167) described previously. ${ }^{20)}$ Plasmid vectors used are as follows: pYES2 from Invitrogen (San Diego, CA, U.S.A.); pT7Blue(R) T-Vector from Novagen (Madison, WI, U.S.A.); pBluescript(r) II SK (-) (pBS II SK (-)) from 
Stratagene (La Jolla, CA, U.S.A.). Polymerase chain reactions (PCRs) were performed with $E x \operatorname{Taq}^{\mathrm{TM}}$ (Takara, Japan). $E$. coli DNA ligase, DNA polymerase I and T4 DNA polymerase were from Takara. Reverse transcriptase was SUPERSCRIPT $^{\mathrm{TM}}$ II (Gibco-BRL, MD, U.S.A.), and Terminal deoxynucleotide transferase (TdT) and RNaseH were from Toyobo (Japan). Synthetic oligo nucleotides for PCR primers were obtained from Nihon Bioservice (Saitama, Japan).

The primer sequences are as follows (one-letter abbreviations are based on the rule of International Union of Biochemistry, IUB):

RACE32: 5'-GACTCGAGTCGACATCGATTTTTTTTTTTTTT-3';

RACE17: 5'-GACTCGAGTCGACATCG-3';

162S: 5'-GAYGGIGGITGGGGIYTICA-3';

463S: 5'-MGICAYATHWSIAARGGIGCITGG-3';

467S: 5'-AARGGIGCITGGCCITTYWSIAC-3';

623A: 5'-CCCAISWICCITMCCAISWICCRTC-3';

711A: 5'-CKRTAYTCICCIARIGCCCADATIGGRAA-3'; CSI-220A: 5'-ATCTTTCCCCATGAGGTTATA-3';

CSI-252A: 5'-CCTGGATGAACTGGCAAGAA-3';

CSI-286A: 5'-CCTTCCTTAGTGACAAGATAAG-3';

CSV-220A: 5'-AACTTTCCCCACGATGAAGTT-3';

CSV-252A: 5'-TCCTGGATGAAGAGGAACTCT-3';

CSV-287A: 5'-CTTTTCTTAGTGATACGACGAT-3';

CSV-486S: 5'-GGAGAAGGACTTGAAGCGGTT-3';

CSV-544S: 5'-GAATCCTGCTGACGTTTTCAT-3';

CSI-N-H: 5'-TTTTAAGCTTAGAATGTGGAGGCTCAAGATCGCGGAG-3' (HindIII is underlined, the start codon is in bold face);

CSI-C-X: 5'-TTCTAGACTAGGCAGGAGATGAATTCAGTG-3' $(X b a \mathrm{I}$ is underlined, the complemented stop codon is in bold face);

CSV-N-B: 5'-GAGAGGATCCGACGATGTGGAGGCTCAAGGTCGCG-3' (BamHI is underlined, the start codon is in bold face);

CSV-C-X: CTTGTGCGCTAACTCGAGTCAAGTCATAGAGTGG-3' (XhoI is underlined, the complemented stop codon is in bold face);

The specific primers designed for $C$. speciosus OSC are summarized in Fig. 1.

RNA and cDNA Preparation Whole parts of 9-weekold plantlets of $C$. speciosus were frozen in liquid nitrogen, and ground into powder in a $1: 1$ mixture of $1 \mathrm{M}$ Tris- $\mathrm{HCl}$ (pH 9) and TE-saturated phenol containing sodium dodecyl sulfate (SDS) (0.5\%). After centrifugation at $15000 \mathrm{rpm}$ for $10 \mathrm{~min}$, the aqueous phase was extracted again with an equal volume of phenol-chloroform-isoamylalcohol $(25: 24: 1)$. The supernatant after centrifugation was subjected to successive precipitation with $\mathrm{EtOH}$ and $\mathrm{LiCl}$ to isolate total RNA. Alternatively, total RNA was isolated using RNeasy ${ }^{\circledR}$ Plant Mini Kit (Qiagen). Poly (A) ${ }^{+}$RNA was purified from the total RNA using a QuickPrep ${ }^{\mathrm{TM}}$ mRNA Purification Kit (Amersham Pharmacia Biotech) according to the manufacturer's protocol. Poly (A) ${ }^{+}$RNA was reverse-transcribed to produce a single-stranded cDNA using $0.5 \mu \mathrm{g}$ of RACE32 primer in a total volume of $20 \mu \mathrm{l}$, followed by $\mathrm{RNaseH}$ treatment. The cDNA mixture was used as a template for core amplification by PCR.

Core Amplification by PCR The oligonucleotide primers used for the amplification of the OSC core region were de-
$A$

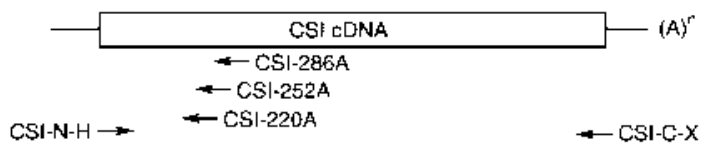

B

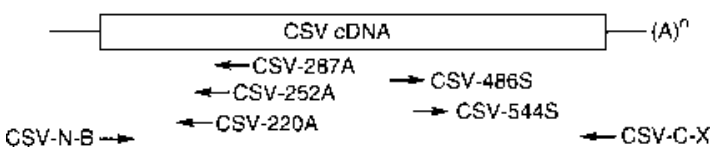

Fig. 1. Location and Direction of CSI (A) and CSV (B) Specific Oligonucleotide Primers

The open boxes represent the coding region, and the solid lines represent the $5^{\prime}$ - and $3^{\prime}$-untranslated regions. The arrows show the location and direction of oligonucleotide primers in relation to the CSI and CSV cDNA above.

signed as described previously. ${ }^{20,21,31)}$ The first PCR with $162 \mathrm{~S}$ and $711 \mathrm{~A}$ primers was performed in a final volume of $100 \mu \mathrm{l}$ for 30 cycles of amplification using a step program $\left(1 \mathrm{~min}\right.$ at $94{ }^{\circ} \mathrm{C}, 2 \mathrm{~min}$ at $42^{\circ} \mathrm{C}$, and $3 \mathrm{~min}$ at $72^{\circ} \mathrm{C}$ ) in the thermal cycler Robocycler Gradient 40 (Stratagene). The PCR product was applied on SUPREC-02 (Takara) to remove the primers, and the volume was adjusted to $100 \mu \mathrm{l}$. A part $(3 \mu \mathrm{l})$ of the solution was used as a template for the second PCR, either with $463 \mathrm{~S}$ and $623 \mathrm{~A}$ or $467 \mathrm{~S}$ and $623 \mathrm{~A}$ primers $(1 \mu \mathrm{g}$ each) under the same conditions as the first PCR. The PCR product of expected size (ca. $0.5 \mathrm{~kb})$ was subcloned into pT7Blue T-vector with a DNA Ligation Kit Ver. 2 (Takara) and propagated in E. coli NovaBlue. DNA sequencing revealed the presence of two types of core fragments (CSI and CSV), which were DIG-labeled following the manufacturer's protocol (Roche Biomedicals).

Library Construction and Screening A cDNA library was constructed from $1.4 \mu \mathrm{g}$ of poly $(\mathrm{A})^{+}$RNA. The firststrand of cDNA was synthesized using reverse transcriptase, and the second-strand was synthesized using E. coli RNase H, E. coli DNA polymerase I, and E. coli DNA ligase. After blunting the termini of cDNA by T4 DNA polymerase, cDNA was ligated to EcoRI adaptors followed by size fractionation ( $>400 \mathrm{bp}$ ) and phosphorylation. Then, cDNA was inserted into the EcoRI site of $\lambda$ ZAPII (Stratagene). The ligated DNA was packaged using a LAMBDA INN in vitro packaging kit (Nippon Gene) and plated on selective $E$. coli XL1-Blue MRF' cells. The resultant library contained $c a$. $1.6 \times 10^{5}$ p.f.u. of independent clones. The DIG-labeled core fragment mixture (CSI and CSV) was used as a probe to screen the cDNA library $\left(5.6 \times 10^{4}\right.$ independent plaques), which was transferred to Hybond $\mathrm{N}^{+}$membrane (Amersham Pharmacia Biotech). The hybridization was carried out at $68^{\circ} \mathrm{C}$ overnight in a solution recommended in the protocol of the DIG Nucleic Acid Detection Kit (Roche Biomedicals). The membranes were washed twice for $5 \mathrm{~min}$ at room temperature in $2 \times \mathrm{SSC} / 0.1 \% \mathrm{SDS}$, followed by washing twice for $15 \mathrm{~min}$ at $68^{\circ} \mathrm{C}$ in $0.1 \times \mathrm{SSC} / 0.1 \% \mathrm{SDS}$. The hybridized DIG-probe was detected according to the manufacturer's protocol. One positive clone was plaque purified, in vivo excised as a phagemid form, and the insert was sequenced. This clone contained $c a$. $1.7 \mathrm{kbp}$ cDNA corresponding to the CSI type.

Cloning of CSI To obtain the full sequence of CSI type, 
stepwise $5^{\prime}$-RACE was performed. For the first step, nested PCR was performed under the same condition as the core amplification. PCRs, first with 162S and 711A and then with $162 \mathrm{~S}$ and 623A primers, yielded 1.4-kb DNA fragment of the CENTER region of both CSI and CSV type cDNAs. Prior to the second step, total RNA was reverse transcribed with the CSI-286A primer, followed by poly (A) tail addition using TdT. The resultant poly (A) tailed cDNA was used as the template for the nested PCR using the primers: 1st, RACE32 and CSI-252A; 2nd, RACE17 and CSI-220A. PCR conditions were the same as in the core amplification, but the annealing temperature for the 2 nd PCR was $54{ }^{\circ} \mathrm{C}$ instead of $42^{\circ} \mathrm{C}$.

Cloning of CSV CSV full-length sequence was obtained as follows. The $3^{\prime}$-end of the CSV cDNA was amplified by nested PCR. PCR was carried out for 30 cycles with a step program $\left(1 \mathrm{~min}\right.$ at $94^{\circ} \mathrm{C}, 2 \mathrm{~min}$ at $56^{\circ} \mathrm{C}$, and $3 \mathrm{~min}$ at $72^{\circ} \mathrm{C}$ ). Primers used were CSV-486S and RACE32 for the 1st PCR, and CSV-544S and RACE17 for the 2nd PCR. 5'-end amplification was performed as in the case for the CSI $5^{\prime}$-end. The CSV-287A primer was used instead of the CSI-286A primer for poly (A) tailed cDNA synthesis. The CSV-252A and CSV-220A primers were used instead of CSI-252A and CSI220A primers for nested PCR.

Functional Expression in Yeast Full-length cDNAs of CSI and CSV were obtained by single-step PCR using a double-stranded cDNA pool as a template. The PCRs with 100 pmol each of primers CSI-N-H and CSI-C-X for CSI, and CSV-N-B and CSV-C-X for CSV were carried out for 30 cycles with a step program $\left(1 \mathrm{~min}\right.$ at $94^{\circ} \mathrm{C}, 2 \mathrm{~min}$ at $62^{\circ} \mathrm{C}$, and $3 \mathrm{~min}$ at $72^{\circ} \mathrm{C}$ ). The amplified DNA was digested with HindIII/XbaI for CSI and BamHI/XhoI for CSV prior to the ligation. Then the PCR products were ligated to the corresponding restriction enzyme sites of a yeast expression vector, pYES2, under the galactose inducible GAL1 promoter. The yeast strain GIL77 was transformed with resultant plasmids pYES2-CSI or pYES2-CSV using Frozen-EZ Yeast Transformation II $^{\mathrm{TM}}$ (Zymo Research). The transformant yeast was grown in synthetic complete medium $(20 \mathrm{ml})$ without uracil (SC-U), supplemented with ergosterol $(20 \mu \mathrm{g} / \mathrm{ml})$, hemin $(13 \mu \mathrm{g} / \mathrm{ml})$, and Tween $80(5 \mu \mathrm{g} / \mathrm{ml})$ at $30^{\circ} \mathrm{C}$ with shaking $(220 \mathrm{rpm})$. After $2 \mathrm{~d}$, cells were collected and resuspended in SC-U without glucose $(20 \mathrm{ml})$, supplemented with ergosterol, hemin, and Tween 80 , and $2 \%$ galactose was added for induction at $30^{\circ} \mathrm{C}$ for $24 \mathrm{~h}$. Cells were collected and resuspended in $0.1 \mathrm{M}$ potassium phosphate, $\mathrm{pH} 7.0$, supplemented with 3\% glucose and hemin and cultured for $24 \mathrm{~h}$ at $30{ }^{\circ} \mathrm{C}$. Cells were collected and refluxed with $5 \mathrm{ml}$ of $20 \%$ $\mathrm{KOH} / 50 \% \mathrm{EtOH}$ at $95^{\circ} \mathrm{C}$ for $1 \mathrm{~h}$. After extraction with the same volume of hexane, the extract was concentrated and applied onto a TLC plate (Merck), which was developed with benzene/acetone (19:1). Spots were detected with phosphomolybdic acid staining. The 4,4-dimethylsterol band was scraped off from the plate, extracted with ethyl acetate, and then subjected to reversed phase HPLC and LC-MS analysis. HPLC analysis was carried out with a TOSOH SC-8010 system employing a SUPER-ODS column $(4.6 \times 200 \mathrm{~mm})$ (TOSOH) with $95 \%$ aqueous $\mathrm{CH}_{3} \mathrm{CN}$ as a solvent (flow rate $1.0 \mathrm{ml} / \mathrm{min}$, detection UV $202 \mathrm{~nm}$ ) at $40^{\circ} \mathrm{C}$.

Liquid Chromatography (LC)-Atmospheric Pressure Chemical Ionization (APCI)-MS Analysis MS spectra were recorded on an LCQ (Thermo Quest) equipped with an HP1100 series LC system (Hewlett Packard) under the same chromatographic conditions used for the HPLC analysis. APCI was applied to detect positive ions of the products.

Cycloartenol: MS m/z $409\left[\mathrm{M}+\mathrm{H}-\mathrm{H}_{2} \mathrm{O}\right]^{+}$, MS/MS (rel. int. \%, precursor ion at $\mathrm{m} / z$ 409) 299 (30), 285 (30), 271 (30), 257 (30), 231 (40), 217 (100), 203 (60), 191 (75), 189 (25).

Lupeol: MS m/z $409\left[\mathrm{M}+\mathrm{H}-\mathrm{H}_{2} \mathrm{O}\right]^{+}, \mathrm{MS} / \mathrm{MS}$ (rel. int. \%, precursor ion at $\mathrm{m} / \mathrm{z} 409) 285$ (70), 271 (100), 259 (75), 257 (75), 231 (25), 229 (35), 215 (30).

Germanicol: MS m/z $409\left[\mathrm{M}+\mathrm{H}-\mathrm{H}_{2} \mathrm{O}\right]^{+}$, MS/MS (rel. int. \%, precursor ion at $\mathrm{m} / z$ 409) 285 (25), 271 (35), 259 (35), 257 (40), 231 (20), 229 (100), 217 (30), 215 (20).

$\beta$-Amyrin: MS m/z $409\left[\mathrm{M}+\mathrm{H}-\mathrm{H}_{2} \mathrm{O}\right]^{+}$, MS/MS (rel. int. $\%$, precursor ion at $m / z 409) 339$ (20), 299 (40), 285 (100), 271 (75), 259 (85), 257 (50), 231 (70), 217 (55), 203 (40), 191 (25), 177 (25).

DNA Sequencing, Computer Analysis of DNA and Protein Sequences The DNA sequence was determined with the dideoxy-chain termination method on double-stranded plasmid (pBS II SK (-)). Sequencing was performed on an automated DNA sequencer model 4000L and 4200L (LICOR Inc., Neb) with the Thermo Sequenase ${ }^{\mathrm{TM}}$ cycle sequencing kit (Amersham Pharmacia Biotech). Sequence information on the RACE fragments was examined based on sequencing at least 6 independent clones. Sequences were analyzed with the DNASIS programs (version 3.7, Hitachi Software Engineering Co., Ltd., Japan). Phylogenetic analysis was carried out with a World Wide Web version (http://spiral.genes.nig.ac.jp/homology/clustalw.shtml) of the CLUSTAL W program ${ }^{32}$ based on a neighbor-joining method. A phylogenic tree was created with the TreeView program (version 1.6.2, freely available from the Taxonomy and Systematics server at the University of Glasgow). The number of bootstrap replications is 1000 . The nucleotide sequence data reported in this paper have been deposited in the DDBJ, EMBL, and GenBank nucleotide sequence databases under the accession numbers AB058507 and AB058508.

\section{RESULTS AND DISCUSSION}

The degenerate primers designed from highly conserved regions of the known OSCs have been successfully applied to PCR-based cloning of novel OSCs. ${ }^{20,21,23,24,28)}$ Combinations of such primers were also used in this study to obtain the target fragments from C. speciosus. Nested PCR gave a 450-bp (CORE) fragment followed by subcloning into a T-vector. DNA sequence analysis of 18 independent clones revealed the presence of two types of DNAs, CSI (14 clones) and CSV (3 clones). The remainder held a hybrid sequence of the two types, and was not investigated further. The deduced product of CSI and CSV showed 75\% mutual identity in sequence, sharing significant $(>50 \%)$ identities with the plant OSCs.

A $\lambda$ ZAPII library harboring cDNAs of $C$. speciosus was screened with a DIG-labeled mixture of CSI and CSV fragments as a probe. One positive clone contained incomplete cDNA corresponding to the CSI fragment, which covered the 3 '-end as well as the poly (A) tail. PCR amplification toward the 3 '-end of a CSV cDNA was attempted by the 'rapid am- 
plification of cDNA ends' (RACE) method. ${ }^{33)}$ The PCR product was shown to contain 646-bp coding and 128-bp non-coding sequences. Stepwise $5^{\prime}$-amplification was subsequently performed by nested PCR to afford a $1.4-\mathrm{kb}$ product (CENTER), which contained an extra $0.9-\mathrm{kb}$ fragment together with the CORE region. HindIII digested the CSI CORE at one site, but left the CSV uncut, allowing the CENTER subclones to be differentiated into 3 CSIs and 2 CSVs. Specific primers were designed for each type based on the CENTER sequence, and subjected to 5'-RACE. Total RNA was reverse transcribed using the CSI-specific primer, CSI286A, followed by poly (A) tail addition. The resultant cDNA mixture was used as a template for nested PCR to give a $0.9-\mathrm{kb}$ product. Sequence analysis revealed the $5^{\prime}$-end sequence, including a putative start codon and a 206-bp noncoding region. Similarly, CSV-specific reverse transcription was conducted. Subsequent nested PCR amplification gave a 0.8-kb product containing 662-bp coding and 79-bp non-coding sequences. Thus, the 'consensus' sequence information on a full-length of cDNA was obtained for both types, CSI and CSV.

For cloning into a yeast expression vector, pYES2, PCR amplification of a full length of each cDNA was performed using the engineered primers containing suitable restriction enzyme sites. Consideration was also given to 'KOZAK' consensus, ${ }^{34)}$ which is adenine 2-bp upstream of the ATG start codon. The template for amplification of full length was a double-stranded cDNA pool prepared for the cDNA library described earlier. Single step PCR using the primer sets successfully afforded $2.3-\mathrm{kb}$ products for both types. For identification of functionally active clones, they were subcloned into the corresponding sites of pYES2 to produce pYES2CSI and pYES2-CSV, respectively. The plasmids were introduced into a yeast mutant, GIL77, which is deficient in lanosterol synthase and accumulates 2,3-oxidosqualene.

Cell extracts of GIL77/pYES2-CSI cultured under galactose induction were subjected to TLC analysis. Four out of eight independent clones gave a spot with an $R f$ value corresponding to 4,4-dimethylsterol, which was not detected in the extracts of a control transformant, GIL77/pYES2. The 4,4dimethylsterol spot on preparative TLC was extracted and analyzed by LC/MS. Profiles of the four samples are essentially identical, showing a single product whose retention time was the same as that of cycloartenol (Fig. 2A). A mass chromatogram indicated the molecular weight to be 426 , and $\mathrm{MS} / \mathrm{MS}$ spectrum of the precursor ion (positive) at $\mathrm{m} / \mathrm{z} 409$ was completely identical with that of cycloartenol (Fig. 2B). The combined results clearly demonstrate that CSI encodes CAS. As cycloartenol could be metabolized into ergosterol in sterol auxotrophic yeast, ${ }^{35)}$ it is normally difficult to detect
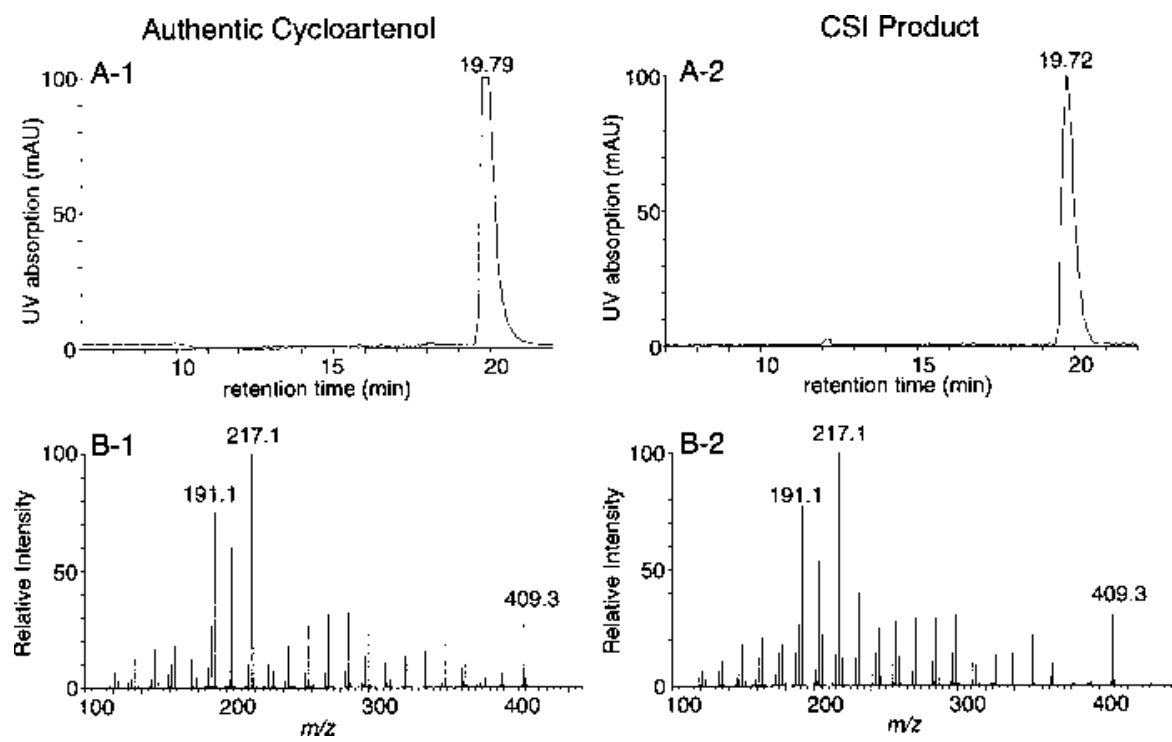

Fig. 2. LC-APCIMS Analysis of CSI Product

(A) HPLC profiles (UV absorption at $202 \mathrm{~nm}$ ) of authentic cycloartenol (A-1) and CSI product (A-2). (B) MS/MS spectra (precursor ion at $m / z 409\left[\mathrm{M}+\mathrm{H}-\mathrm{H}_{2} \mathrm{O}\right]^{+}$) of authentic cycloartenol (B-1) and CSI product (B-2).
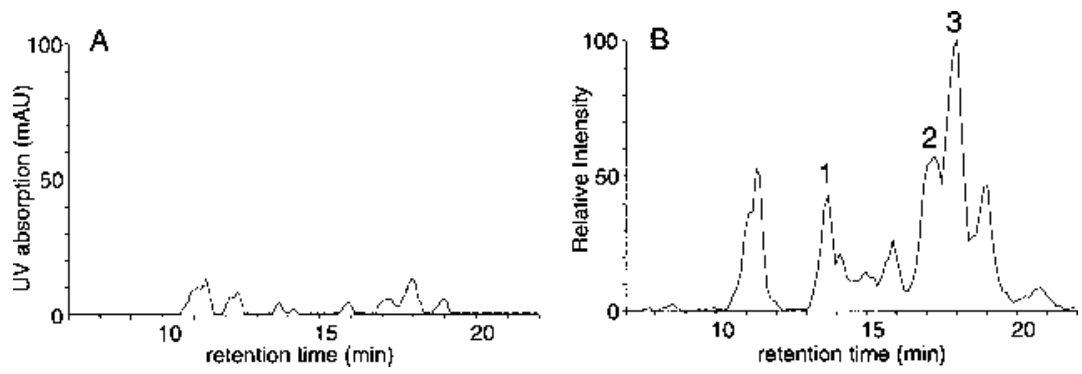

Fig. 3. LC-APCI-MS Analysis of CSV Product

(A) HPLC profile (UV absorption at $202 \mathrm{~nm}$ ). (B) Mass chromatogram $\left(\mathrm{m} / \mathrm{z} 409\left[\mathrm{M}+\mathrm{H}-\mathrm{H}_{2} \mathrm{O}\right]^{+}\right.$). Components of several peaks (peak 1 at $13.73 \mathrm{~min}$; lupeol, peak 2 at $17.26 \mathrm{~min}$; germanicol, and peak 3 at $18.01 \mathrm{~min} ; \beta$-amyrin) were determined by LC-APCI-MS analysis. 
the in vivo accumulation of cycloartenol produced by heterologously expressed CAS. Functional analysis of CAS in our previous studies relied on an in vitro enzyme assay using cell free extracts of recombinant yeast. ${ }^{21)}$ In the present study, however, the cell extract gave a distinct spot on TLC that corresponds to cycloartenol, along with several spots that are not observed in the transformants with cDNAs of triterpene synthases. The latter are likely to be cycloartenol metabolites of the host yeast on the way to ergosterol.

Under the same analytical conditions, three out of eight recombinants of GIL77/pYES2-CSV gave a 4,4-dimethylsterol spot with significantly less intensity. An HPLC profile with absorbance at $202 \mathrm{~nm}$ gave multiple peaks (Fig. 3A), and the same pattern was also observed in the mass chromatogram at $\mathrm{m} / \mathrm{z} 409$ (Fig. 3B). This is the typical case encountered for multifunctional triterpene synthases. ${ }^{24,27-30)}$ By comparison of MS/MS fragmentation patterns of $m / z 409$ with those of authentic samples, lupeol, germanicol, and $\beta$-amyrin were identified as CSV products. The other triterpenes were not further characterized because of difficulty in HPLC separation and limited availability of standard samples. However, it is clearly concluded that this clone is a 'multi-product' synthase rather than a 'product specific' one.

The functionally characterized recombinants were subjected to DNA sequencing. All of the clones from the respective types (CSI type: 4 clones; CSV type: 3 clones) were revealed to be essentially identical and in agreement with the foregoing 'consensus' sequences. Both cDNAs (CSI type: CSOSC1; CSV type: CSOSC2) consist of a 2280-bp coding sequence, encoding 759 amino acids with $74 \%$ mutual identity. Their overall sequence identity with the known plant OSCs was 55 to $80 \%$. Phylogenetic tree analysis of the deduced products suggested that these proteins, along with the putative OSCs from monocot plants, occupy distinct positions (Fig. 4). Although monocot OSCs may constitute a separate branch from the CASs and triterpene synthases of dicot origin, further phylogenic evaluation awaits more cloning ex- amples from monocot plants.

C. speciosus is a rich source of steroidal metabolites; however, so far no cyclic triterpenes have been reported. The identification of a multifunctional triterpene synthase cDNA implies that this plant produces a mixture of triterpenes, although they have not been reported, most probably due to their low productivity.

In conclusion, we have succeeded in the molecular cloning of cDNAs encoding CAS and multifunctional triterpene synthase from C. speciosus. This is the first example ${ }^{36)}$ of the functional expression of OSC from monocot plants. Our initial question was if there are separate cycloartenol pools for the biosynthesis of phytosterols and steroidal glycosides. The

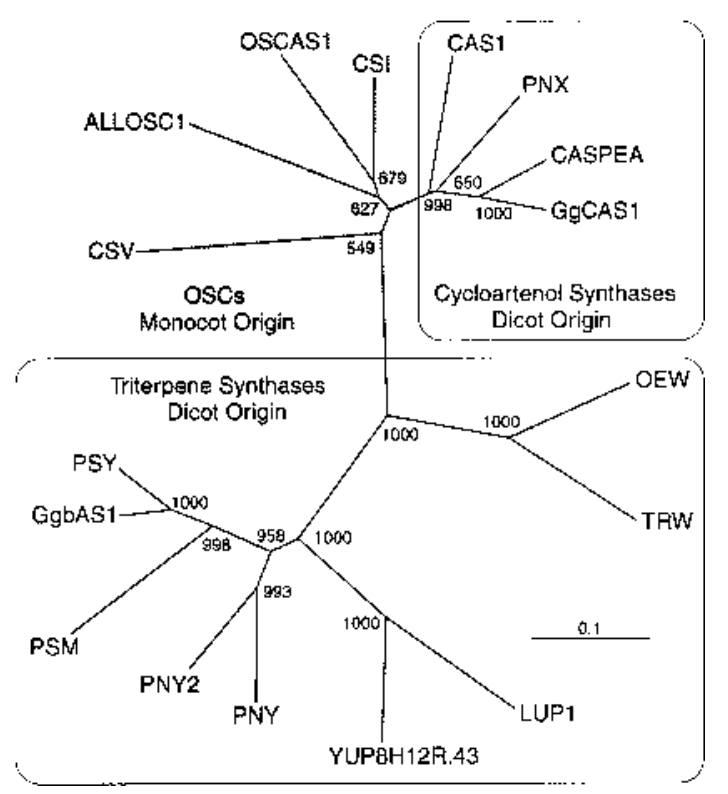

Fig. 4. Phylogenetic Tree of Selected Plant OSCs

See text for abbreviations of clone designations. The indicated scale represents 0.1 amino acid substitution per site. The bootstrap values are shown at the branch point.

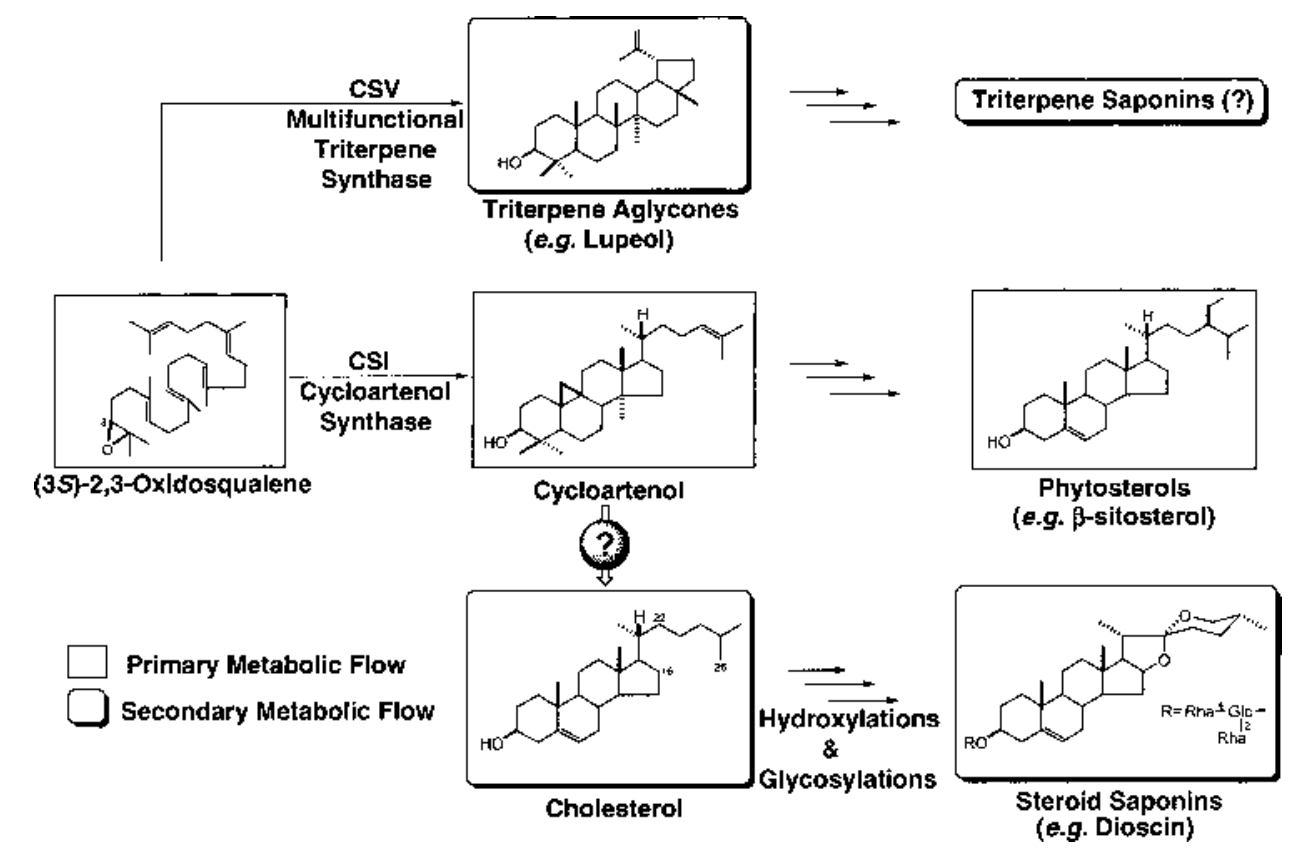

Fig. 5. Proposed Biosynthetic Pathways for Steroid Saponins and Related Metabolites in C. speciosus 
present study indicates the expression of CAS from a single pool of mRNA, implying that the metabolic branch point between the two pathways exists further downstream of the biosynthetic routes, such as at the side chain modification stage (Fig. 5). In most cases, plant secondary metabolism is under developmental control by which relevant pathways are localized to a particular organelle. In vitro cultured plantlet of $C$. speciosus, however, accumulates steroidal glycosides in whole parts, including leaf, root, and stem. ${ }^{7)}$ A cDNA encoding furostanol glycoside $26-O$ - $\beta$-glucosidase was successfully cloned $^{8)}$ from the cDNA pools from the cDNA pools from the plantlet. We believe that a whole pathway of the glycosides is actively expressed, but apparently more dedicated genes to this pathway are to be characterized. Another possibility is that CAS constitutes a family of genes that are identical at the mature mRNA level. Genomic organization of CAS and subsequent transcriptional analysis are in progress to clarify the problem.

Acknowledgements This work was supported by a Grant-in-Aid for Scientific Research from the Ministry of Education, Science, Sports, Culture and Technology of Japan (11771316) (to K.I.). Further financial support was obtained from the Japan Society for the Promotion of Science (Research for the Future Program, Grant no. JSPSRFTF96I00302) (to Y.E.).

\section{REFERENCES AND NOTES}

1) Mahato S. B., Ganguly A. N., Sahu N. P., Phytochemistry, 21, 959978 (1982).

2) Inoue K., Kobayashi S., Noguchi H., Sankawa U., Ebizuka Y., Nat. Med., 49, 336-339 (1995).

3) Marker R. E., Wagner R. B., Ulshafer P. R., Wittbecker E. L., Goldsmith, D. P. J., Ruof C. H., J. Am. Chem. Soc., 69, 2167-2230 (1947).

4) Akhila A., Gupta M. M., J. Plant Physiol., 130, 285-290 (1987).

5) Inoue K., Shimomura K., Kobayashi S., Sankawa U., Ebizuka Y., Phytochemistry, 41, 725-727 (1996).

6) Inoue K., Ebizuka Y., FEBS Lett., 378, 157-160 (1996).

7) Inoue K., Ebizuka Y., Adv. Exp. Med. Biol., 404, 57-69 (1996).

8) Inoue K., Shibuya M., Yamamoto K., Ebizuka Y., FEBS Lett., 389, 273-277 (1996)

9) Ichinose K., You S., Kawano N., Hayashi K., Yao X.-S., Ebizuka Y., Phytochemistry, 51, 599-603 (1999).

10) Joly R. A., Bonner J., Benett R. D., Heftmann E., Phytochemistry, 8, $857-859$ (1969)

11) Stohs S. J., Kaul B., Staba E. J., Phytochemistry, 8, 1679-1686 (1969).

12) Joly R. A., Bonner J., Benett R. D., Heftmann E., Phytochemistry, 8,
1709-1711 (1969).

13) Stohs S. J., Sabatka J. J., Rosenburg H., Phytochemistry, 13, 2145 2148 (1974)

14) Tal B., Tamir I., Rokem J. S., Goldberg I., Biochem. J., 219, 619-624 (1984)

15) Bennett R. D., Heftmann E., Joly R. A., Phytochemistry, 9, 349-353 (1970).

16) Tomita Y., Uomori A., J. Chem. Soc. Chem. Commun., 1971, 284.

17) Bergenstråhle A., Borgå P., Jonsson L. M. V., Phytochemistry, 41, 155-161 (1996).

18) Abe I., Rohmer M., Prestwich G. D., Chem. Rev., 93, 2189-2206 (1993).

19) Corey E. J., Matsuda S. P. T., Bartel B., Proc. Natl. Acad. Sci. U.S.A., 90, 11628-11632 (1993).

20) Kushiro T., Shibuya M., Ebizuka Y., Eur. J. Biochem., 256, 238-244 (1998).

21) Morita M., Shibuya M., Lee M.-S., Sankawa U., Ebizuka Y., Biol. Pharm. Bull., 20, 770-775 (1997).

22) Hayashi H., Hirooka N., Ikeshiro Y., Kushiro T., Morita M., Shibuya M., Ebizuka Y., Biol. Pharm. Bull., 23, 231-234 (2000).

23) Kushiro T., Shibuya M., Ebizuka Y., "Towards Natural Medicine Research in the 21st Century," Excerpta Medica International Congress Series 1157, ed. by Ageta H., Aimi N., Ebizuka Y., Fujita T., Honda G., Elsevier, 1998, pp. 421-428.

24) Morita M., Shibuya M., Kushiro T., Masuda K., Ebizuka Y., Eur. J. Biochem., 267, 3453-3460 (2000).

25) Hayashi H., Huang P., Kirakosyan A., Inoue K., Hirooka N., Ikeshiro Y., Kushiro T., Shibuya M., Ebizuka Y., Biol. Pharm. Bull., 24, 912916 (2001).

26) Shibuya M., Zhang H., Endo A., Shishikura K., Kushiro T., Ebizuka Y., Eur. J. Biochem., 266, 302-307 (1999).

27) Herrera J. B. R., Bartel B., Wilson W. K., Matsuda S. P. T., Phytochemistry, 49, 1905-1911 (1998).

28) Segura M. J., Meyer M. M., Matsuda S. P. T., Org. Lett., 2, 2257 2259 (2000)

29) Kushiro T., Shibuya M., Masuda K., Ebizuka Y., Tetrahedron Lett., 41, $7705-7710$ (2000)

30) Husselstein-Muller T., Schaller H., Benveniste P., Plant Mol. Biol., 45, $75-92$ (2001).

31) You S., Kawano N., Ichinose K., Yao X.-S., Ebizuka Y., Plant Biotechnol., 16, 311-314 (1999).

32) Thompson J. D., Higgins D. G., Gibson T. J., Nucleic Acids Res., 22, 4673-4680 (1994).

33) Frohman M. A., Dush M. K., Martin M. K., Proc. Natl. Acad. Sci. U.S.A., 85, 8998-9002 (1988).

34) Kozak M., Nucleic Acids Res., 12, 857-872 (1984)

35) Venkatramesh M., Nes W. D., Arch. Biochem. Biophys., 324, 189-199 (1995).

36) After the submission of this manuscript, a proceeding ${ }^{37)}$ describing cDNAs, AsbAS1 and $A s C S 1$, encoding $\beta$-amyrin synthase and putative CAS, respectively, from a monocot plant, Avena strigosa, appeared.

37) Haralampidis K., Bryan G., Qi X., Papadopoulou K., Bakht S., Melton R., Osbourn A., Proc. Natl. Acad. Sci. U.S.A., 98, 13431-13436 (2001) 